\title{
MANAGEMENT OF GINGIVAL HYPERPIGMENTATION USING DIODE LASER ABLATION VERSUS ROTATORY ABRASION INSTRUMENT
}

\author{
Rania Farouk Abdulmaguid*
}

\begin{abstract}
Fifteen cases suffering from gingival hyper pigmentation from MSA outpatient clinic, are treated in a split mouth design where half the arch is treated using a rotary abrasion while the other is treated using diode laser. Pain was assessed using visual pain analogue during the first week following depigmentation procedure, melanin pigmentation was assessed using melanin index which was evaluated at base line, one week, one month, 3 months and 6 months follow up periods. Aim of the study is to compare rotary abrasion and diode laser concerning pain and recurrence of gingival hyper pigmentation throughout the follow up period. Results: Both techniques showed minimal pain and discomfort, with minor pigmentation recurrence with rotatory abrasion at the 6 months follow up period. In addition to less bleeding with better field visualization during the procedure using diode laser in addition to reduced pain and discomfort.
\end{abstract}

\section{INTRODUCTION}

Aspects of dental treatment have changed to achieve acceptable gingival aesthetics while addressing biologic and functional problems due to increase aesthetics awareness and demand by patients ${ }^{(1)}$. Coral pink is the normal color of the gingiva which is considered as the normal physiologic color with variations of melanin pigmentation.

The melanin gingival pigmentation is considered to play a protective role in gingival inflammation process, and is commonly seen in dark-skinned individuals ${ }^{(2)}$. Melanin, powerful cation chelator, is a nonhemoglobin-derived brown pigment and is the most common endogenous pigment present in the body that is produced by melanocytes ${ }^{(3)}$.

A wide range of lesions and conditions may cause gingival discoloration, which may include physiologic etiology as racial pigmentation, systemic illness manifestations as Addison's disease, to malignant neoplasms as melanoma and Kaposi's sarcoma. Therefore, it is necessary to determine the cause of pigmentation before commencing any depigmentation treatment ${ }^{(4)}$. The presence of physiologically melanin hyperpigmented gingiva does not present as a medical problem, but rather

\footnotetext{
* Lecturer at Oral Medicine and Periodontology Department Faculty of Dentistry MSA University.
} 
it may present as an aesthetic one with patients complaining of having black gums, which may be more aggravated in patients with a gummy smile or excessive gingival display while talking or smiling ${ }^{(5)}$.

Roshni \& Nandakumar in 2005 classified different gingival depigmentation methods as ${ }^{(5)}$ :

1) Methods aimed at removing the pigmented gingiva:

a) SURGICAL METHODS:

i) Scalpel surgical technique,

ii) Bur abrasion method,

iii) Electrosurgery,

iv) Cryosurgery,

v) Lasers,

vi) Radiosurgery.

b) CHEMICAL METHODS.

2) Methods aimed at masking the pigmented gingiva:

a) Free gingival graft.

b) Acellular dermal matrix allograft.

Stone abrasion techniques for gingival depigmentation utilize high speed rotary instruments with large round or straight burs and copious saline irrigation to de-epithelize heavily pigmented gingival areas. Such techniques are simple and relatively effortless without the need for complicated or expensive armamentarium. Stone abrasion is associated with complications such as pain, bleeding and difficulty in controlling the depth of de-epithelization ${ }^{(6)}$.

Lasers have been used in dentistry since the beginning of the 1980s ${ }^{(7)}$. Different lasers such as carbon dioxide (CO2) laser, Nd:YAG laser, semiconductor diode laser, argon laser, Er:YAG laser and Er,Cr:YSGG laser have been reported as effective and reliable method with minimal postoperative discomfort and faster wound healing for depigmentation procedure ${ }^{(8)}$. The diode laser is a solid-state semiconductor laser that typically uses a combination of Gallium (Ga), Arsenide (Ar), and other elements, such as Aluminum (Al) and Indium (In), to change electrical energy into light energy ${ }^{(9)}$.

Dental laser energy has an affinity for different tissue components. The $810-\mathrm{nm}$ diode laser has energy and wavelength characteristics that specially target the soft tissues. It has an affinity for hemoglobin and melanin, therefore it is more efficient and better equipped to address deeper soft tissue problems ${ }^{(10)}$.

\section{MATERIALS AND METHODS}

Fifteen patients who were concerned with their aesthetics 10 males and 5 females, were treated with two different techniques in a split mouth design at the Department of Periodontics, Faculty of Dentistry, MSA University, Cairo, Egypt. In the present study, the patients who were included complained of 'black gum' related to the maxillary anterior region, and they were keen to get it corrected. The criteria for the selection of the patients in the present study were the aesthetic considerations in periodontally healthy patients. The patients who were treated in this study were enrolled in the period within the years of 2016 to 2017.

\section{Patient selection and methods of evaluation:}

Based on the degree of pigmentation as assessed by Melanin pigmentation index (Takashi et al. ${ }^{(11)}$, the degree of melanin pigmentation was determined by melanin pigmentation index based on the following scoring system:

\section{Melanin pigmentation index}

- Score 0: No pigmentation

- Score 1: Solitary unit(s) of pigmentation in papillary gingiva without extension between neighboring solitary units 
- Score 2: Formation of continuous ribbon extending from neighboring solitary units.

Detailed medical history was recorded before starting treatment to exclude pregnancy, systemic diseases, malignancy, medications, uncontrolled diabetes.

Detailed extra oral/intra oral examinations were carried out, which included the evaluation of the skin pigmentation and the perioral pigmented lesions . Before undertaking any of the depigmentation procedures, all the patients underwent a full mouth scaling, following which oral hygiene instructions were given to them.

\section{Visual pain analogue scale:}

The VAS ${ }^{(12)}$ was used to measure the intensity of pain experienced during and after treatment. The VAS consisted of a horizontal line $100 \mathrm{~mm}$ long $(1-10 \mathrm{~cm})$, anchored at the left end by the descriptor "no pain" and at the right end by "unbearable pain." The patient placed a mark to coincide with the level of pain. The distance of this point, in millimeters, from the left end of the scale was recorded and used as the VAS score. Scores were calculated as: $0=$ no pain; 0.1 to $3.0 \mathrm{~cm}(1$ to $30 \mathrm{~mm})=$ mild pain; 3.1 to $6.0 \mathrm{~cm}(31$ to $60 \mathrm{~mm})=$ moderate pain; 6.1 to $10 \mathrm{~cm}(61$ to $100 \mathrm{~mm})=$ severe pain .

\section{Smile line evaluation:}

Smile line was evaluated for each patient as all the study patients smile line ranged from high smile line (class II) and average smile line (class III) .

The classification of smile line was taken according to Liebert's classification ${ }^{(13)}$ :

- Class 1: VERY HIGH SMILE LINE $->2 \mathrm{~mm}$ of the marginal gingiva is visible or $>2 \mathrm{~mm}$ apical to the CEJ is Visible.

- Class2: HIGH SMILE LINE - Between 0 - 2 $\mathrm{mm}$ of marginal gingiva is visible or between 0 and $2 \mathrm{~mm}$ apical to the CEJ is visible.
- Class3: AVERAGE SMILE LINE- Only gingival embrasures are visible.

- Class4: LOW SMILE LINE- Gingival embrasures and $\mathrm{CEJ}$ are not visible.

\section{Gingival depigmentation using gingival abrasion technique:}

For the gingival abrasion technique, a high speed doughnut shaped coarse diamond points were attached to the high speed rotary handpiece with a water connection and a local anesthesia injection (2\% Lidocaine Hydrochloride with 1,00,000 adrenaline which was manufactured by Novocol, Canada) were used. A periodontal dressing was used to cover the wound.

A spray of saline was constantly directed on the rotating points during their use. An accelerated speed ensures a smooth, rapid operation, while the stream of saline provides a temperature control and it prevents clogging of the burs. The procedure was carried out until the underlying soft connective tissue was exposed. Then, the area was mopped with a wet gauze to stop the bleeding. Once the bleeding had subsided, a periodontal dressing was placed over the operated area. Post-operative instructions were given, to avoid eating hot and spicy food stuffs to avoid the bleeding. The patients were asked to take analgesics if there was any pain

\section{Gingival depigmentation using diode laser:}

The other half of the anterior maxilla was treated using diode laser (figure 1-3). Before starting the procedure, the area which had to be operated was infiltrated with a local anesthetic agent $(2 \%$ Lidocaine Hydrochloride with 1,00,000 adrenaline which was manufactured by Novocol, Canada) for standardization. After this, retraction of upper lip was made by Minoseta retractor. We used diode laser Sirona $810 \mathrm{~nm}$ wavelength, made by Sirona Dental Systems GmbH with 3W CW, $400 \mu m$ fiber, in sweeping motion. The diode laser was set and 
safety protection such as use of specific room and wearing specific eye glasses was applied. Then we used the laser tip on the surface of the tissues in contact mode. In this process, almost three minutes after laser therapy, the de-epithelialization of gingiva occurred, then we scratched a piece of wet sterile gauze on the de- epithelialized surface and detached the epithelium, after which the laser was irradiated on the denuded area for coagulation of this area. The lack of bleeding during and following the procedure (figures 4,5 ) was considered as a

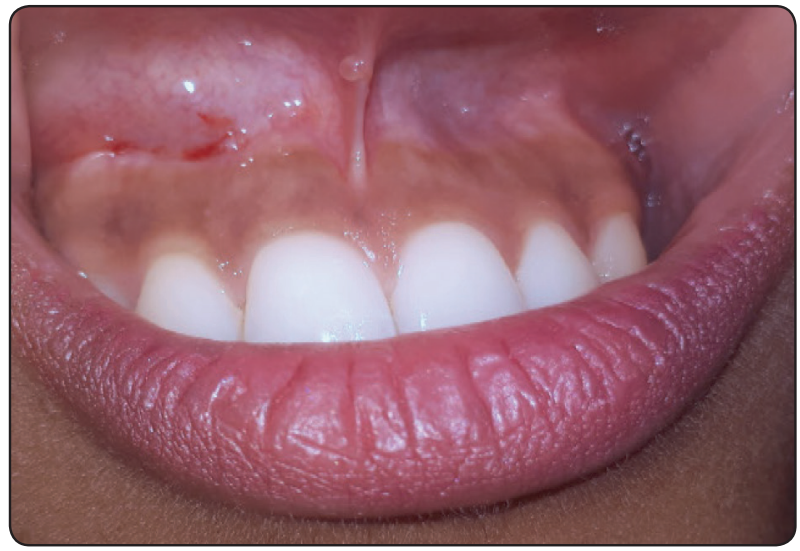

Fig. (1) patient before depigmentation showing bilateral pigmentation with score 2Melanin index

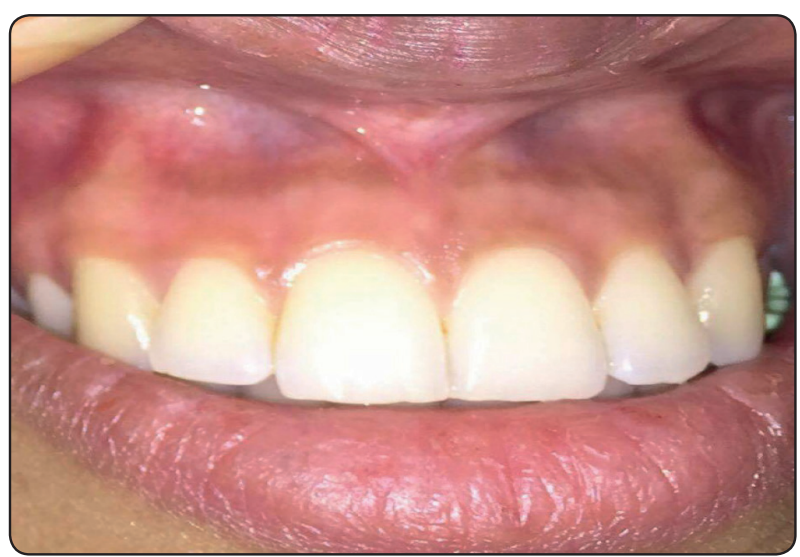

Fig. (3) at 6 months follow up showing partial recurrence of pigmentation at the left side (gingival abrasion site) time saving factor and added more convenience for the operator and the patient, all the wounds were covered using Coe pack for both study sites for the sake of standardization.

The patients were recalled on the 1st week, first month, 3rd month and 6th month post operatively. Patients were instructed to avoid smoking and eating of hot and spicy foods for the first 24 hours and were discharged from dental office to perform normal daily activity.

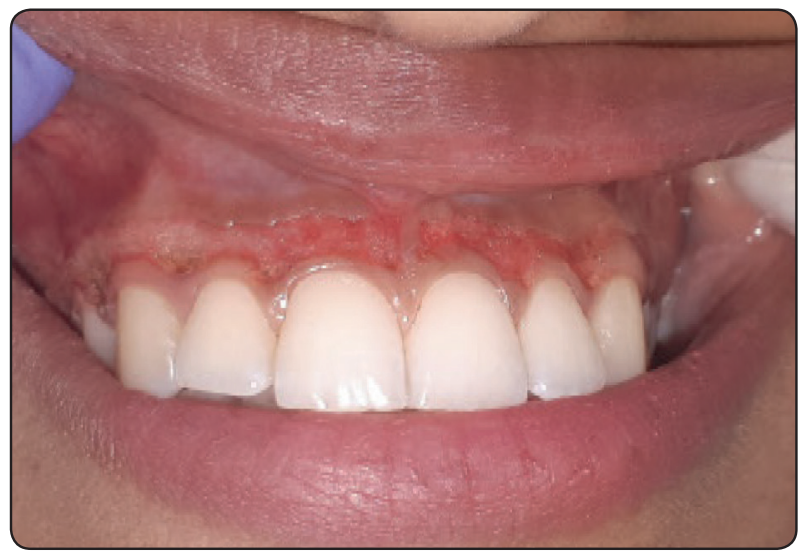

Fig. (2) a split mouth depigmentation using laser on the Right side of the patient while gingival abrasion on the left side.

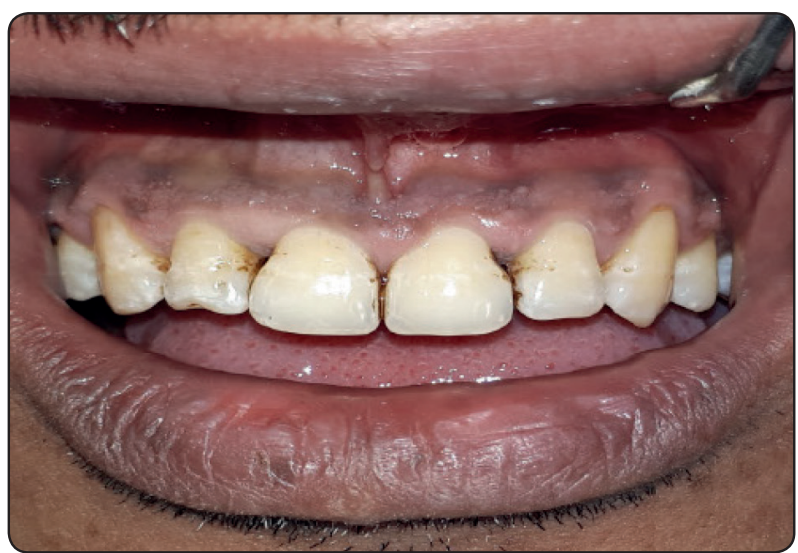

Fig. (4) patient before depigmentation showing bilateral pigmentation with score 2Melanin index 


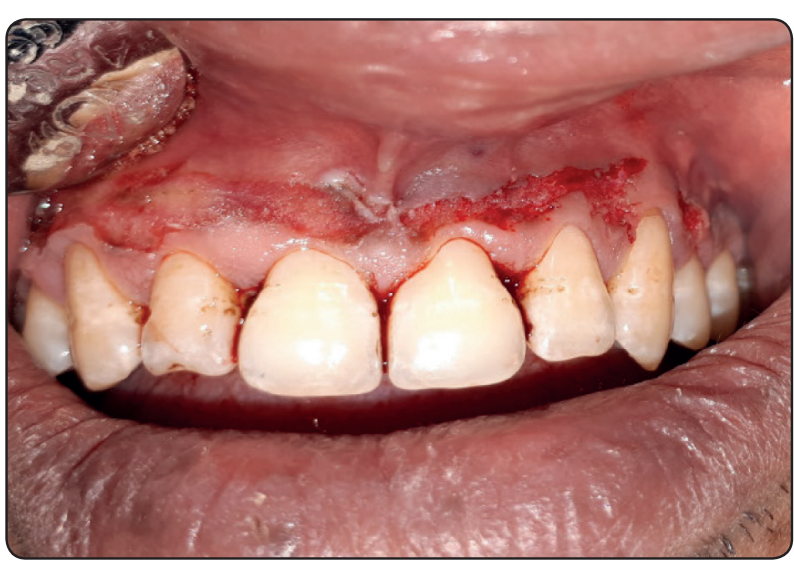

Fig. (5) a split mouth depigmentation using laser on the Right side of the patient without bleeding while gingival abrasion on the left side showing post-surgical bleeding

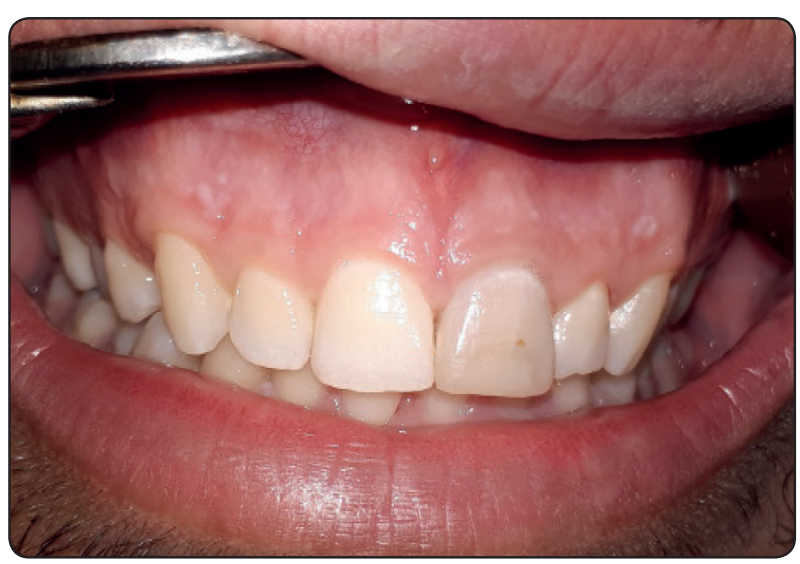

Fig. (7) At 6 months post treatment with no recurrence of pigmentation

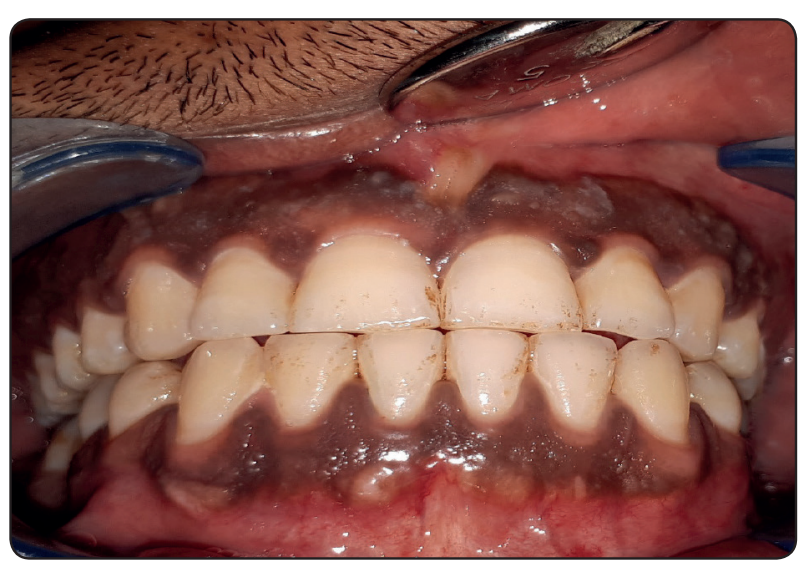

Fig. (6) patient before depigmentation showing bilateral pigmentation with score 2 Melanin index

\section{RESULTS}

\section{Evaluation of Pigmentation}

The results (table 1) of this study were statistically analyzed using one-way analysis of variance (ANOVA). Comparisons were conducted between the different periods of the study and the baseline readings. The analysis was as follows:

TABLE (1) Tabulated values of the depigmentation procedure

\begin{tabular}{|c|c|c|c|c|c|c|c|c|c|}
\hline \multirow{2}{*}{ No. } & \multirow{2}{*}{ Baseline } & \multicolumn{2}{|c|}{7 days } & \multicolumn{2}{c|}{1 month } & \multicolumn{2}{c|}{3 month } & \multicolumn{2}{c|}{6 month } \\
\cline { 3 - 9 } & & $\mathrm{A}$ & $\mathrm{L}$ & $\mathrm{A}$ & $\mathrm{L}$ & $\mathrm{A}$ & $\mathrm{L}$ & $\mathrm{A}$ & $\mathrm{L}$ \\
\hline 1 & 3 & 0 & 0 & 0 & 0 & 1 & 0 & 1 & 0 \\
\hline 2 & 2 & 0 & 0 & 0 & 0 & 1 & 0 & 1 & 1 \\
\hline 3 & 2 & 0 & 0 & 0 & 0 & 0 & 0 & 1 & 0 \\
\hline 4 & 3 & 0 & 0 & 0 & 0 & 1 & 1 & 1 & 0 \\
\hline 5 & 1 & 0 & 0 & 0 & 0 & 0 & 0 & 0 & 0 \\
\hline 6 & 3 & 0 & 0 & 0 & 0 & 0 & 0 & 1 & 0 \\
\hline 7 & 2 & 0 & 0 & 0 & 0 & 0 & 0 & 0 & 0 \\
\hline 8 & 2 & 0 & 0 & 0 & 0 & 0 & 0 & 1 & 1 \\
\hline 9 & 3 & 0 & 0 & 0 & 0 & 0 & 0 & 1 & 0 \\
\hline 10 & 1 & 0 & 0 & 0 & 0 & 0 & 0 & 1 & 0 \\
\hline 11 & 2 & 0 & 0 & 0 & 0 & 0 & 0 & 1 & 0 \\
\hline 12 & 2 & 0 & 0 & 0 & 0 & 0 & 0 & 1 & 0 \\
\hline 13 & 2 & 0 & 0 & 0 & 0 & 1 & 0 & 1 & 0 \\
\hline 14 & 2 & 0 & 0 & 0 & 0 & 1 & 0 & 1 & 0 \\
\hline 15 & 2 & 0 & 0 & 0 & 0 & 1 & 0 & 1 & 0 \\
\hline
\end{tabular}


Baseline vs Abrasion and Laser at different follow-up intervals

Abrasion and Laser techniques proved to be very effective as depigmentation techniques with sustained results throughout the follow-up period of the study. When comparing the melanin index baseline readings and the follow-up for both techniques at 3-months and 6-months the results were extremely significant with a p-value of $<0.001$.

\section{Abrasion vs Laser at 3-months:}

When comparing the results of both techniques at 3-months there was no statistical difference between them with a p-value of $>0.05$. Both techniques maintained the results attained following the surgical intervention.

\section{Abrasion vs Laser at 6-months:}

Although both techniques maintained the depigmentation of the gingiva, some recurrence of pigmentation was evidenced in the abrasion group (not to the original baseline levels) but not in the laser group. When comparing the results of both Table (2) Visual pain analogue scale techniques at 6-months there was high statistical difference between them with a p-value of $<0.001$.

\section{Intra group analysis at different follow-up inter-} vals:

When comparing the results of the abrasion technique at 3- and 6-months there was statistical difference with a p-value of $<0.05$, this may be the result of the recurrence of pigmentation that was evidenced after 6 months.

On the other hand, there was no statistical difference between the follow-up intervals with the laser technique with a p-value of $>0.05$.

\section{Evaluation of Pain:}

The analysis of pain (table 2) related to the procedure utilized was measured using visual pain analogue scale for seven days following the surgical intervention. In both groups, no pain was registered in the study population from the fourth day onward for both techniques. Statistical analysis was conducted for the first three days using paired-t test for each day separately for both techniques.

\begin{tabular}{|c|c|c|c|c|c|c|c|c|c|c|c|c|c|c|}
\hline & \multicolumn{2}{|c|}{ Day 1} & \multicolumn{2}{|c|}{ Day 2} & \multicolumn{2}{|c|}{ Day 3} & \multicolumn{2}{|c|}{ Day 4} & \multicolumn{2}{|c|}{ Day 5} & \multicolumn{2}{|c|}{ Day 6} & \multicolumn{2}{|c|}{ Day 7} \\
\hline & A & $\mathrm{L}$ & A & $\mathrm{L}$ & A & $\mathrm{L}$ & A & $\mathrm{L}$ & A & $\mathrm{L}$ & A & $\mathrm{L}$ & A & L \\
\hline 1 & 1 & 2 & 1 & 1 & 1 & 1 & 0 & 0 & 0 & 0 & 0 & 0 & 0 & 0 \\
\hline 2 & 1 & 1 & 1 & 1 & 1 & 1 & 0 & 0 & 0 & 0 & 0 & 0 & 0 & 0 \\
\hline 3 & 2 & 2 & 1 & 1 & 1 & 1 & 0 & 0 & 0 & 0 & 0 & 0 & 0 & 0 \\
\hline 4 & 1 & 1 & 1 & 1 & 0 & 0 & 0 & 0 & 0 & 0 & 0 & 0 & 0 & 0 \\
\hline 5 & 1 & 1 & 0 & 0 & 0 & 0 & 0 & 0 & 0 & 0 & 0 & 0 & 0 & 0 \\
\hline 6 & 1 & 1 & 1 & 1 & 0 & 0 & 0 & 0 & 0 & 0 & 0 & 0 & 0 & 0 \\
\hline 7 & 1 & 1 & 0 & 1 & 0 & 0 & 0 & 0 & 0 & 0 & 0 & 0 & 0 & 0 \\
\hline 8 & 1 & 1 & 1 & 1 & 1 & 1 & 0 & 0 & 0 & 0 & 0 & 0 & 0 & 0 \\
\hline 9 & 1 & 1 & 0 & 1 & 0 & 0 & 0 & 0 & 0 & 0 & 0 & 0 & 0 & 0 \\
\hline 10 & 2 & 2 & 1 & 1 & 0 & 1 & 0 & 0 & 0 & 0 & 0 & 0 & 0 & 0 \\
\hline 11 & 1 & 1 & 0 & 1 & 0 & 0 & 0 & 0 & 0 & 0 & 0 & 0 & 0 & 0 \\
\hline 12 & 1 & 1 & 0 & 1 & 0 & 0 & 0 & 0 & 0 & 0 & 0 & 0 & 0 & 0 \\
\hline 13 & 1 & 1 & 0 & 1 & 0 & 0 & 0 & 0 & 0 & 0 & 0 & 0 & 0 & 0 \\
\hline 14 & 1 & 1 & 0 & 1 & 0 & 0 & 0 & 0 & 0 & 0 & 0 & 0 & 0 & 0 \\
\hline 15 & 1 & 1 & 0 & 1 & 0 & 1 & 0 & 0 & 0 & 0 & 0 & 0 & 0 & 0 \\
\hline
\end{tabular}


Day one showed no significant difference between the techniques with a p-value of 0.6383 . On day two there was highly significant difference between the two techniques with a p-value of 0.0041 , more pain was registered with the abrasion technique although the pain was mild. On the third day, there was no significant difference between the two techniques with a p-value of 0.4560 .

\section{DISCUSSION}

Bur Abrasion Technique was demonstrated to be an effective way for the elimination of gingival pigmentation with relative ease but was associated with bleeding, inability to determine the depth of tissue removal, and mild pain post-operatively ${ }^{(6)}$.

Laser ablation was shown to be one of the most efficient, convenient and reliable techniques for gingival depigmentation (14). Gingival depigmentation performed in this study was performed by diode laser Sirona $810 \mathrm{~nm}$ wavelength, made by Sirona Dental Systems GmbH with 3W CW as it has near to the maximum absorption for melanin and hemoglobin, additionally in comparison with Er: YAG, diode laser has the advantage of effective and safe application, being able to prevent bleeding, with limitation of postoperative inflammation and pain and a favorable gingival healing ${ }^{(15)}$.

In the present study, the patients found that, as compared to the rotary technique, the diode laser management site was well - tolerated. All the patients were satisfied with the results and had high acceptance for the procedure as a simple and effective technique as they have undergone the rotary and laser depigmentation (split mouth design).

Although both techniques maintained gingival depigmentation, some recurrence of pigmentation was evidenced in the abrasion group (not to the original baseline levels). This was in agreement with the results of Kumar et al ${ }^{(16)}$ whom demonstrated increased pain and inflammatory changes associated with rotary techniques, and the reappearance of the pigmentation which was observed after 30 days following the treatment. Also, a study by Ginwalla et al (17) reported repigmentation after 33 days, which was also in accordance with studies done by Dummett and Bolden ${ }^{(18)}$ and Pal et al ${ }^{(19)}$.

According to Bandish ${ }^{(20)}$ melanin repigmentation was not observed following depigmentation procedures due to either "migration" of the melanocytes did not occur or the melanocytes that migrated were in an inactive state due to the inhibition of their tyrosinase system. Repigmentation may also be attributed to the melanocytes which are left during surgery as stated by Ginwalla et al ${ }^{(17)}$.

Good tactile sensation during the laser procedure was done while the laser was in a contact mode, using the contact mode with water spray was reported to achieve precise irradiation, good tactile sensation and reduced thermal effect in a clean operating environment ${ }^{(21-23)}$, in addition to that complete deepithelization requires that the instruments are used in this mode, to get the best control of the laser beam without harming the neighboring teeth, and alveolar bone ${ }^{(24)}$,the reliability of this method was proved also in the present study.

Obtaining dry surgical field during laser procedure is possible due to absence of bleeding after laser treatment which can be attributed to the property of lasers that seals blood vessels through the coagulation effect of laser which assists in obtaining a relatively dry surgical field ${ }^{(25)}$. Laser is absorbed by soft tissue pigments, thus making it an excellent hemostatic agent ${ }^{(21)}$.

Successful treatment following laser ablation of hyper-pigmented areas was evident in the present study by the uneventful healing of the gingiva and complete regeneration resulting in a healthy pink firm appearance. These findings confirm and extend the previous data on the successful application of laser techniques for the treatment of gingival hyperpigmentation ${ }^{(26)}$. Photo modulation 
effects of laser were shown to help in stimulating the fibroblasts, angiogenesis and accelerating the lymphatic flow, which promotes repair and regeneration. In addition, the bactericidal effect of laser, which is owed to the production of reactive oxygen species, may also add to the rapid healing in a relatively sterile environment ${ }^{(27,28)}$.

\section{CONCLUSION}

The use of a diode laser was demonstrated to be a safe, reliable and effective treatment modality as compared to rotary technique to provide maximum aesthetics with reduced patients discomfort during the treatment of gingival hyper pigmentation, a more follow up period is recommended in further studies to test the long-term sustainability of depigmentation in both techniques.

The present study showed that Abrasion and Laser techniques proved to be very effective as depigmentation techniques with sustained results throughout the follow-up of the study.

The large variation in time of repigmentation may be related to the technique used and the race of the patient ${ }^{(29)}$.

\section{REFERENCES}

1. Gulati N, Dutt P, Gupta N, Tyagi P. Gingival Pigmentation: Revisited. J AdvMed Dent Scie Res 2016; 4: 48-57.

2. Eid HA, Syed S, Soliman AN. The role of gingival melanin pigmentation in inflammation of gingiva, based on genetic analysis. J Int Oral Health 2013; 5: 1-7.

3. Nakamura Y, Funato A, Wakabayashi H, Matsumoto K. A study on the removal of the melanin pigmentation of dog gingiva by $\mathrm{CO} 2$ laser irradiation. J Clin Laser Med Surg 1992; 10: 41-6.

4. Jonathan A Ship, Martin S Greenberg, Michael Glick (ed). Burket's oral medicine: Pigmented lesions of the oral mucosa. 10th ed. Hamilton Ontario: BC Decker Inc; 2003.

5. Roshni T, Nandakumar K. Anterior esthetic gingival depigmentation and crown lengthening: Report of a case. J Contemp Dent Pract. 2005; 15: 139-147.
6. Farnoosh AA. Treatment of gingival pigmentation and discoloration for esthetic purpose. Int J Periodont Rest Dent. 1990; 10: 312-319.

7. Esen E, Haytac MC, Oz IA, Erdoğan O, Karsli ED. Gingival melanin pigmentation and its treatment with $\mathrm{CO} 2$ laser. Oral Surg Oral Med Oral Pathol Oral Radiol Endod 2004; 98:522-527.

8. Suthprasertporn S. Treatment of gingival melanin hyperpigmentation by Er,Cr:YSGG laser: Report of 2 cases. Thai J Periodontol 2007; 1:46-55.

9. Lagdive S, Doshi Y, Marawar PP. Management of gingival hyperpigmentation using surgical blade and diode laser therapy: A comparative study. J Oral Laser Appl 2009; 9:41-47.

10. Miyasaki MA. Shedding light on the soft tissue laser. Signature 2004; 11:11-13.

11. Takashi H, Tanaka K, Ojima M, Yuuki K. Association of melanin pigmentation in the gingiva of children with parents who smoke. Pediatrics. 2005; 116: 186-190.

12. Huskisson EC. Measurement of pain. J Rheumatol. 1982; 9:768-769.

13. Liebert MF, Fouque Dervelle C, Santani A, Dilier FL, Monnet Corti V, Glise JM, et al. Smile line and periodontal visibility. Perio 2004;1:17-25.

14. Berk G, Attici K, Berk N. Treatment of gingival pigmentation with Er, Cr: YSGG laser. J Oral Laser Applications. 2005;5:249-253.

15. Giannelli M, Formigli L, Bani D. Comparative evaluation of photoablative efficacy of erbium: yttrium-aluminiumgarnet and diode laser for the treatment of gingival hyperpigmentation. A randomized split-mouth clinical trial. J Periodontol. 2014;85(4):554-561.

16. Kumar S, Bhat GS, Bhat KM. Comparative Evaluation of Gingival Depigmentation using Tetrafluoroethane Cryosurgery and Gingival Abrasion Technique: Two Years Follow Up. Journal of Clinical and Diagnostic Research. 2013, Vol-7: 389-394.

17. Ginwalla TM, Gomes BC, Verma BRR. Surgical removal of gingival pigmentation (A preliminary study). J Indian Dent Assoc. 1966; 38: 147

18. Dummett CO, Bolden TE. Post-surgical clinical repigmentation of the gingiva. Oral Surg Oral Med Oral Pathol Oral Radiol Endod. 1963; 16: 353-65. 
19. TK Pal, KK Kapoor, CC Parel, K Mukherjee. Gingival melanin pigmentation - A study on its removal for esthetic. J Indian Soc of Periodontology. 1994; (special Issue) 3: $52-54$.

20. Bandish LK. Pertinent considerations in oral pigmentation. Brit Dent J. 1985 Mar 9; 158 (5): 158

21. Rosa DS, Aranha AC, Eduardo Cde P, Aoki A. Esthetic treatment of gingival melanin hyperpigmentation with Er:YAG laser: short-term clinical observations and patient follow-up. J Periodontol. 2007;78(10):2018-25.

22. Tal H, Oegiesser D, Tal M. Gingival depigmentation by erbium:YAG laser: Clinical observations and patient response. J Periodontol. 2003;74:1660-1667.

23. Ishii S, Aoki A, Kawashima Y, Watanabe H, Ishikawa I. Application of an Er: YAG laser to remove gingival melanin hyperpigmentation - Treatment procedure and clinical evaluation. J Jpn Soc Laser Dent. 2002;13:89-96.

24. Giannelli M, Formigli L, Lasagni M, Bani D. A new thermographic and fluorescent method for tuning photoablative laser removal of the gingival epithelium in patients with chronic periodontitis and hyperpigmentation. Photomed Laser Surg. 2013;31(5):212-218.

25. Atsawasuwan P, Greethong K, Nimmanon V. Treatment of gingival hyperpigmentation for esthetic purposes by Nd: YAG laser: Report of 4 cases. J Periodontol. 2000; 71:315-321.

26. Khakar M, Kapoor R, Jayakumar, Padmalatha O, Varghese SS, Sankari M. Advantages of 980nm diode laser treatment in the management of gingival pigmentation J Laser Dent. 2011;19(3):283-285.

27. Ozbayrak S, Dumlu A, Ercalik-Yalcinkaya S.Treatment of melanin-pigmented gingiva and oral mucosa by $\mathrm{CO} 2$ laser. Oral Surg Oral Med Oral Pathol Oral Radiol Endod. 2000

28. Singh V, Gillyar, Kumar S, Bhat M. Comparative evaluation of gingival depigmentation by diode laser and cryosurgery using tetrafluoroethane: 18 month follow up. CAP. 2012;2(3): 129-133.

29. Gupta G. Management of Gingival Hyperpigmentation by Semiconductor Diode Laser. J Cutan Aesthet Surg. 2011; Vol 4: 208 -210 